\title{
ORDINARY PSEUDOREPRESENTATIONS AND MODULAR FORMS
}

\author{
PRESTON WAKE AND CARL WANG-ERICKSON
}

(Communicated by Romyar T. Sharifi)

\begin{abstract}
In this note, we observe that the techniques of our paper "Pseudomodularity and Iwasawa theory" can be used to provide a new proof of some of the residually reducible modularity lifting results of Skinner and Wiles. In these cases, we have found that a deformation ring of ordinary pseudorepresentations is equal to the Eisenstein local component of a Hida Hecke algebra. We also show that Vandiver's conjecture implies Sharifi's conjecture.
\end{abstract}

\section{INTRODUCTION}

The key technical innovation behind our previous work WWE15 was our definition of an ordinary 2-dimensional pseudorepresentation of $G_{\mathbb{Q}}=\operatorname{Gal}(\overline{\mathbb{Q}} / \mathbb{Q})$. Using this notion, we were able to study ordinary Galois deformations in the case where the residual representation is reducible. In particular, we constructed a universal ordinary pseudodeformation ring $R_{\bar{D}}^{\text {ord }}$ with residual pseudorepresentation $\bar{D}$. We also showed that the Galois action on the Eisenstein part of the cohomology of modular curves gives rise to an ordinary pseudorepresentation valued in the Eisenstein component $\mathfrak{H}$ of the ordinary Hecke algebra. Studying deformations of ordinary pseudorepresentations, we showed that if Greenberg's conjecture holds, then certain characteristic 0 localizations $\mathfrak{H}_{\mathfrak{p}}$ of $\mathfrak{H}$ are Gorenstein. Under the same assumption, we also proved an isomorphism $\left(R_{\bar{D}}^{\text {ord }}\right)_{\mathfrak{p}} \stackrel{\sim}{\rightarrow} \mathfrak{H}_{\mathfrak{p}}$.

In this note, we show that the methods of WWE15 can be extended to the whole Eisenstein component $\mathfrak{H}$, provided that we make stronger assumptions on class groups. Namely, we have to assume that the plus-part $X^{+}$of the Iwasawa class group of the relevant cyclotomic field vanishes. When the tame level $N$ is 1 , then this is known as Vandiver's conjecture. When $N>1$, there are examples where $X^{+} \neq 0$, but it is still often the case that $X^{+}=0$. Assuming $X^{+}=0$, we get an isomorphism $R_{\bar{D}}^{\text {ord }} \stackrel{\sim}{\rightarrow} \mathfrak{H}$ (Theorem 4.2.8). As a consequence, we have a new technique to establish the residually reducible ordinary modularity theorem of Skinner and Wiles [SW99] over $\mathbb{Q}$, in some cases (Theorem 5.2.4). We also derive new results on Gorensteinness of Hecke algebras (Corollary 5.1.1) and prove new results toward Sharifi's conjecture (Corollary 5.1.2). In particular, we prove that $\mathfrak{H}$ is Gorenstein when $X^{+}=0$, an implication that was known previously only after assuming Sharifi's conjecture Wak15b]. Previous partial results in this direction by Skinner-Wiles [SW97] and Ohta Oht05] require much stronger conditions on class groups.

Received by the editors October 7, 2015, and, in revised form, October 17, 2015, November 28, 2015, July 10, 2016, December 16, 2016, and January 13, 2017.

2010 Mathematics Subject Classification. 11F33, 11F80, 11R23. 
As well as proving these new results, we review the most novel parts of [WWE15]. In this way, this note may serve as an introduction to [WWE15.

1.1. Ordinary pseudorepresentations. A 2-dimensional pseudorepresentation of $G_{\mathbb{Q}}$ with values in a ring $A$ is the data of two functions $\{\operatorname{tr}$, det $\}$ that satisfy conditions as if they were the trace and determinant of a representation $G_{\mathbb{Q}} \rightarrow$ $\mathrm{GL}_{2}(A)$. The (fine) moduli of pseudorepresentations may be thought of as the coarse moduli of Galois representations produced by geometric invariant theory [WE15, Thm. A]. In this respect, our results suggest that coarse moduli rings of Galois representations are most naturally comparable with Hecke algebras. Indeed, most previous $R=T$ theorems have been established where $R$ is a deformation ring for a residually irreducible Galois representation, in which case the fine and coarse moduli of Galois representations are identical.

The ordinary condition is somewhat subtle when applied to pseudorepresentations. For example, if one thinks about the case when $A$ is a field, a representation $\rho: G_{\mathbb{Q}} \rightarrow \mathrm{GL}_{2}(A)$ is defined to be ordinary when $\left.\rho\right|_{G_{\mathbb{Q} p}}$ is reducible with a twistunramified quotient. While $\left\{\left.\operatorname{tr} \rho\right|_{G_{\mathbb{Q} p}}\right.$, $\left.\left.\operatorname{det} \rho\right|_{G_{\mathbb{Q} p}}\right\}$ knows nothing about which of the two Jordan-Hölder factors is the quotient, $D_{\rho}=\{\operatorname{tr} \rho, \operatorname{det} \rho\}$ can often distinguish them. This allows for the definition of an ordinary pseudorepresentation of $G_{\mathbb{Q}}$, which we extend to non-field coefficients. We overview this and other background from [WWE15] in $\S \S 2$ and 3.

1.2. Outline of the proof. The étale cohomology of compactified modular curves defines a $G_{\mathbb{Q}^{-}}$-module $H$ over the cuspidal quotient $\mathfrak{h}$ of $\mathfrak{H}$. However, $H$ is a representation (i.e., locally free $\mathfrak{h}$-module) if and only if $\mathfrak{h}$ is Gorenstein, which is not always true. Nonetheless, $H$ always induces an ordinary $\mathfrak{h}$-valued pseudorepresentation deforming the residual pseudorepresentation $\bar{D}$. This pseudorepresentation extends to $\mathfrak{H}$, resulting in a surjection $R_{\bar{D}}^{\text {ord }} \rightarrow \mathfrak{H}$.

This map is naturally a morphism of augmented $\Lambda$-algebras, where $\Lambda$ is an Iwasawa algebra. The augmentation ideals

$$
\mathcal{I}:=\operatorname{ker}(\mathfrak{H} \rightarrow \Lambda), \quad \mathcal{J}:=\operatorname{ker}\left(R_{\bar{D}}^{\text {ord }} \rightarrow \Lambda\right)
$$

correspond to the Eisenstein family of $\Lambda$-adic modular forms and the reducible locus of Galois representations, respectively. We can show that certain Iwasawa class groups surject onto $\mathcal{J} / \mathcal{J}^{2}$, which is the cotangent module relative to the reducible family. The Vandiver-type assumption $X^{+}=0$ is used to show that one of the relevant Iwasawa class groups is cyclic. Using a version of Wiles's numerical criterion [Wil95, Appendix], with the class groups playing the role of Wiles's $\eta$, we can show that this forces $R_{\bar{D}}^{\text {ord }} \rightarrow \mathfrak{H}$ to be an isomorphism.

One aspect of this proof is that we are able to control a "cotangent space" $\mathcal{J} / \mathcal{J}^{2}$ of a pseudodeformation ring in terms of Galois cohomology. We use Galois cohomology groups with coefficients in $\Lambda$. Such control is critical to proving $R=T$ theorems in the residually irreducible case, and $R=T$ theorems for pseudorepresentations were lacking because this control was not as available. In our situation, the relevant Galois cohomology is determined by class groups. 


\section{Background: Iwasawa theory and Hecke algebras}

This section is a brief synopsis of $\S \S 2,3$, and 6 of WWE15. We overview background information from Iwasawa theory and ordinary $\Lambda$-adic Hecke algebras and modular forms.

2.1. Iwasawa algebra and Iwasawa modules. We review $\S 2$ of [WWE15].

Let $p \geq 5$ be a prime number, and let $N$ be an integer such that $p \nmid N \phi(N)$. Let

$$
\theta:(\mathbb{Z} / N p \mathbb{Z})^{\times} \rightarrow \overline{\mathbb{Q}}_{p}^{\times}
$$

be an even character. Let $\chi=\omega^{-1} \theta$, where

$$
\omega:(\mathbb{Z} / N p \mathbb{Z})^{\times} \rightarrow(\mathbb{Z} / p \mathbb{Z})^{\times} \rightarrow \mathbb{Z}_{p}^{\times}
$$

is the Teichmüller character. Our assumption on $N$ implies that each of these characters is a Teichmüller lift of a character valued in a field extension $\mathbb{F}$ of $\mathbb{F}_{p}$. By abuse of notation, we also use $\theta, \chi, \omega$ to refer to these characters.

We assume that $\theta$ satisfies the following conditions:

(a) $\theta$ is primitive,

(b) if $\left.\chi\right|_{(\mathbb{Z} / p \mathbb{Z})^{\times}}=1$, then $\left.\chi\right|_{(\mathbb{Z} / N \mathbb{Z}) \times}(p) \neq 1$, and

(c) if $N=1$, then $\theta \neq \omega^{2}$.

A subscript $\theta$ or $\chi$ on a module refers to the eigenspace for an action of $(\mathbb{Z} / N p \mathbb{Z})^{\times}$. A superscript \pm will denote the \pm 1 -eigenspace for complex conjugation. Let $S$ denote the set of primes dividing $N p$ along with the infinite place, and let $G_{\mathbb{Q}, S}$ be the unramified outside $S$ Galois group. We fix a decomposition group $G_{p} \subset G_{\mathbb{Q}, S}$ and let $I_{p} \subset G_{p}$ denote the inertia subgroup. Let $\kappa_{\text {cyc }}$ denote the $p$-adic cyclotomic character.

Fix a system $\left(\zeta_{N p^{r}}\right)$ of primitive $N p^{r}$-th roots of unity such that $\zeta_{N p^{r+1}}^{p}=\zeta_{N p^{r}}$ for all $r$. Let $\mathbb{Q}_{\infty}=\mathbb{Q}\left(\zeta_{N p^{\infty}}\right)$ and let $\Gamma=\operatorname{Gal}\left(\mathbb{Q}_{\infty} / \mathbb{Q}\left(\zeta_{N p}\right)\right)$.

Let $\mathrm{Cl}\left(\mathbb{Q}\left(\zeta_{N p^{r}}\right)\right)$ be the class group, and let

$$
X=\lim _{\longleftarrow} \mathrm{Cl}\left(\mathbb{Q}\left(\zeta_{N p^{r}}\right)\right)\{p\} .
$$

There is action of $\Gamma$ on $X$. By class field theory, $X=\operatorname{Gal}\left(L / \mathbb{Q}_{\infty}\right)$ where $L$ is the maximal pro- $p$, abelian, unramified extension of $\mathbb{Q}_{\infty}$. A closely related object is $\mathfrak{X}=\operatorname{Gal}\left(M / \mathbb{Q}_{\infty}\right)$, where $M$ is the maximal pro- $p$ abelian extension unramified outside $N p$.

Let $\mathbb{Z}_{p, N}=\lim \mathbb{Z} / N p^{r} \mathbb{Z}$. Let $\Lambda_{\theta}=\mathbb{Z}_{p} \llbracket \mathbb{Z}_{p, N}^{\times} \rrbracket_{\theta}$. We write $\Lambda$ for $\Lambda_{\theta}$ when $\theta$ is implicit. This $\Lambda$ is a local component of the semilocal ring $\mathbb{Z}_{p} \llbracket \mathbb{Z}_{p, N}^{\times} \rrbracket$ and is abstractly isomorphic to $\mathcal{O} \llbracket \Gamma \rrbracket \simeq \mathcal{O} \llbracket T \rrbracket$, where $\mathcal{O}$ is the extension of $\mathbb{Z}_{p}$ generated by the values of $\theta$. Notice that the action on $\left(\zeta_{N p^{r}}\right)$ gives an isomorphism $\Gamma \simeq$ $\operatorname{ker}\left(\mathbb{Z}_{p}^{\times} \rightarrow(\mathbb{Z} / p \mathbb{Z})^{\times}\right)$.

Let $M \mapsto M^{\#}$ and $M \mapsto M(r)$ be the functors on $\mathbb{Z}_{p} \llbracket \mathbb{Z}_{p, N}^{\times} \rrbracket$-modules as defined in Wak15a, §2.1.3]. Namely, $M^{\#}=M(r)=M$ as $\mathbb{Z}_{p}$-modules, but $\gamma \in \mathbb{Z}_{p, N}^{\times}$acts on $M^{\#}(\operatorname{resp} . M(r))$ as $\gamma^{-1}\left(\operatorname{resp} . \kappa_{\text {cyc }}^{r}(\gamma) \gamma\right)$ acts on $M$. We sometimes, especially when using duality, are forced to consider $\mathbb{Z}_{p} \llbracket \mathbb{Z}_{p, N}^{\times} \rrbracket$-modules with characters other than $\theta$, but we use these functors to make the actions factor through $\Lambda$ so we can treat all modules uniformly.

We define $\xi_{\chi} \in \Lambda$ to be a generator of the principal ideal $\operatorname{Char}_{\Lambda}\left(X_{\chi}(1)\right)$. By the Iwasawa Main Conjecture, it may be chosen to be a power series associated to a Kubota-Leopoldt $p$-adic $L$-function. 
Consider the $\Lambda$-valued character $\langle-\rangle: G_{\mathbb{Q}, S} \rightarrow \Gamma \subset \Lambda^{\times}$, where $G_{\mathbb{Q}, S} \rightarrow \Gamma$ is the quotient map. We define $\Lambda\left[G_{\mathbb{Q}, S}\right]$-modules $\Lambda^{\langle-\rangle}$and $\Lambda^{\#}$ to be $\Lambda$ with $G_{\mathbb{Q}, S}$ acting by $\langle-\rangle$ and $\langle-\rangle^{-1}$, respectively.

2.2. Duality and consequences. We review some relevant parts of $\S 6$ of WWE15. To compare conditions on various class groups, we use the following $\Lambda$-adic version of Poitou-Tate duality. It is a generalization of WWE15, Prop. 6.2.1].

Here, $K$ is a number field, and $U$ is an open dense subset of $\operatorname{Spec}\left(O_{K}[1 / p]\right)$. The compactly supported cohomology $H_{(c)}^{\bullet}(U,-)$ is defined to be the cohomology of

$$
\text { Cone }\left(C^{\bullet}(U,-) \rightarrow \bigoplus_{v \notin U} C^{\bullet}\left(K_{v},-\right)\right),
$$

where $C^{\bullet}(U,-)$ and $C^{\bullet}\left(K_{v},-\right)$ are the standard complexes that compute Galois cohomology.

Proposition 2.2.1. Let $T$ be a finitely generated projective $\Lambda$-module equipped with a continuous action of $G_{K}$, unramified at places outside $U$, and let $M$ be a finitely generated $\Lambda$-module. Then there is a quasi-isomorphism

$$
\mathrm{R} \Gamma_{(c)}\left(U, T \otimes_{\Lambda} M\right) \stackrel{\sim}{\longrightarrow} \operatorname{RHom}_{\Lambda}\left(\mathrm{R} \Gamma\left(U, T^{*}(1)\right), M\right)[-3]
$$

that is functorial in $M$. There is a similar quasi-isomorphism when $\mathrm{R} \Gamma_{(c)}$ and $\mathrm{R} \Gamma$ are swapped, i.e.,

$$
\mathrm{R} \Gamma\left(U, T \otimes_{\Lambda} M\right) \stackrel{\sim}{\longrightarrow} \operatorname{RHom}_{\Lambda}\left(\mathrm{R}_{(c)}\left(U, T^{*}(1)\right), M\right)[-3] .
$$

Here $T^{*}$ is the dual representation $\operatorname{Hom}(T, \Lambda)$.

Proof. We prove the first quasi-isomorphism. The proof of the second is similar.

For the case where $M=\Lambda$, see [Nek06, Prop. 5.4.3, p. 99] or [FK12, §1.6.12]. Then we have quasi-isomorphisms

$$
\begin{aligned}
\mathrm{R}_{(c)}(U, T) \otimes_{\Lambda}^{\mathbb{L}} M & \stackrel{\sim}{\rightarrow} \operatorname{RHom}_{\Lambda}\left(\mathrm{R} \Gamma\left(U, T^{*}(1)\right), \Lambda\right)[-3] \otimes_{\Lambda}^{\mathbb{L}} M \\
& \stackrel{\sim}{\longrightarrow} \operatorname{RHom}_{\Lambda}\left(\mathrm{R} \Gamma\left(U, T^{*}(1)\right), M\right)[-3],
\end{aligned}
$$

where the first comes from the $M=\Lambda$ case, and the second is standard (for example Wei94, Exer. 10.8.3]). To prove the proposition, we are reduced to producing a quasi-isomorphism

$$
\mathrm{R}_{(c)}(U, T) \otimes_{\Lambda}^{\mathbb{L}} M \stackrel{\sim}{\longrightarrow} \mathrm{R} \Gamma_{(c)}\left(U, T \otimes_{\Lambda} M\right) .
$$

This follows from [LS13, Prop. 3.1.3] (and its compactly supported analog, which, as remarked in the proof of Proposition 4.1.1 of [LS13, can be established similarly).

The proposition yields spectral sequences with second pages

$$
\begin{aligned}
& E_{2}^{i, j}=\operatorname{Ext}_{\Lambda}^{i}\left(H^{3-j}\left(U, T^{*}(1)\right), M\right) \Longrightarrow H_{(c)}^{i+j}\left(U, T \otimes_{\Lambda} M\right), \\
& E_{2}^{i, j}=\operatorname{Ext}_{\Lambda}^{i}\left(H_{(c)}^{3-j}\left(U, T^{*}(1)\right), M\right) \Longrightarrow H^{i+j}\left(U, T \otimes_{\Lambda} M\right) .
\end{aligned}
$$

These spectral sequences are functorial in $M$.

We record the influence of the assumption that $X_{\theta}=0$. In the proof, we make use of the following lemma on the structure of $\Lambda$-modules. 
Lemma 2.2.5. Let $M$ be a finitely generated $\Lambda$-module. Say that $M$ is type 0 if $M$ is free, type 1 if $M$ is torsion and has projective dimension 1 , and type 2 if $M$ is finite. Then $M$ is type $i$ if and only if $\operatorname{Ext}_{\Lambda}^{j}(M, \Lambda)=0$ for all $j \neq i$. Moreover, $M$ is type 1 if and only if $M$ is torsion and has no non-zero finite submodule.

Proof. See [Jan89, §3].

The following is well-known to experts.

Proposition 2.2.6. $X_{\theta}=0$ if and only if $\mathfrak{X}_{\chi^{-1}}^{\#}(1)$ is a free $\Lambda$-module of rank 1 .

Proof. As in [WWE15, Cor. 6.3.1], we have $X_{\theta}=H^{2}\left(\mathbb{Z}[1 / N p], \Lambda^{\#}(1)\right)$. Since $\mathfrak{X}$ is the Pontryagin dual of $H^{1}\left(\mathbb{Z}[1 / N p], \mathbb{Q}_{p} / \mathbb{Z}_{p}\right)$, (classical) Poitou-Tate duality implies that

$$
\mathfrak{X}=\lim _{\longleftarrow} H_{(c)}^{2}\left(\mathbb{Z}\left[1 / N p, \zeta_{N p^{r}}\right], \mathbb{Z}_{p}(1)\right) .
$$

We can then deduce that $\mathfrak{X}_{\chi^{-1}}^{\#}(1)=H_{(c)}^{2}\left(\mathbb{Z}[1 / N p], \Lambda^{\langle-\rangle}\right)$, as in WWE15, Cor. 6.1.3].

Analyzing spectral sequence (2.2.4) above with $T=\Lambda^{\langle-\rangle}$and $M=\Lambda$, we see that $E_{2}^{i, j}=0$ for cohomological dimension reasons unless $i, j \in\{0,1,2\}$. We find that

$$
\operatorname{Ext}_{\Lambda}^{1}\left(\mathfrak{X}_{\chi^{-1}}^{\#}(1), \Lambda\right)=X_{\theta}, \quad \operatorname{Ext}_{\Lambda}^{2}\left(\mathfrak{X}_{\chi^{-1}}^{\#}(1), \Lambda\right)=0 .
$$

Then $\mathfrak{X}_{\chi^{-1}}^{\#}(1)$ is a free $\Lambda$-module if and only if $X_{\theta}=0$ by Lemma 2.2.5.

The fact that the rank is then 1 follows from class field theory and Iwasawa's theorem. Indeed, class field theory implies that there is an exact sequence

$$
0 \rightarrow U_{\chi^{-1}}^{\#}(1) \rightarrow \mathfrak{X}_{\chi^{-1}}^{\#}(1) \rightarrow X_{\chi^{-1}}^{\#}(1) \rightarrow 0,
$$

where $U$ is an Iwasawa local unit group at $p$, and Iwasawa's theorem implies that $U_{\chi^{-1}}^{\#}(1)$ is free of rank 1 over $\Lambda$ (see [WWE15, $\S 2.1$ ] and the references given there; note that there is no contribution from the local units at primes dividing $N$ because $\theta$ is primitive). Since $X_{\chi^{-1}}^{\#}(1)$ is $\Lambda$-torsion, this implies that $\mathfrak{X}_{\chi^{-1}}^{\#}(1)$ has rank 1.

2.3. Hecke algebras. We review $\S 3$ of WWE15. Let

$$
\tilde{H}^{\prime}=\lim _{\longleftarrow} H^{1}\left(Y_{1}\left(N p^{r}\right), \mathbb{Z}_{p}\right)_{\theta}^{\text {ord }}, H^{\prime}=\lim _{\longleftarrow} H^{1}\left(X_{1}\left(N p^{r}\right), \mathbb{Z}_{p}\right)_{\theta}^{\text {ord }},
$$

where the subscript $\theta$ denotes the eigenspace for the diamond operators. Let $\mathfrak{H}^{\prime}$ and $\mathfrak{h}^{\prime}$ denote the Hida Hecke algebras acting on $\tilde{H}^{\prime}$ and $H^{\prime}$, respectively. There is a unique maximal ideal of $\mathfrak{H}^{\prime}$ containing the Eisenstein ideal for $\theta$; let $\mathfrak{H}$ and $\mathfrak{h}$ be the localizations of $\mathfrak{H}^{\prime}$ and $\mathfrak{h}^{\prime}$ at the Eisenstein maximal ideal, and let $\tilde{H}=\tilde{H}^{\prime} \otimes_{\mathfrak{H}^{\prime}} \mathfrak{H}$ and $H=H^{\prime} \otimes_{\mathfrak{h}} \mathfrak{h}$. Let $\mathcal{I} \subset \mathfrak{H}$ be the Eisenstein ideal, and let $I \subset \mathfrak{h}$ be the image of $\mathcal{I}$.

By Hida theory, each of $\tilde{H}, H, \mathfrak{H}$, and $\mathfrak{h}$ is finite and flat over $\Lambda$. There are also canonical isomorphisms of $\mathfrak{H}$-modules $\mathfrak{H} / \mathcal{I} \cong \Lambda, \mathfrak{h} / I \cong \Lambda / \xi_{\chi}$, and $\mathcal{I} \cong I$ (see WWE15, Prop. 3.2.5]), making $\mathfrak{H}$ an augmented $\Lambda$-algebra.

\section{ORdinary PSEUdorepresentations}

We define ordinary pseudorepresentations and show that they are representable by an ordinary pseudodeformation ring $R_{\bar{D}}^{\text {ord }}$, recapitulating results of [WWE15]. In particular, we will review background on pseudorepresentations, Cayley-Hamilton algebras, and generalized matrix algebras from $\S 5$ of WWE15. 
We highlight the following important points:

- The definition is "not local", in the sense that it does not have the form " $D: G_{\mathbb{Q}, S} \rightarrow A$ is ordinary if $\left.D\right|_{G_{p}}$ is ordinary".

- When $A$ is a field, we can say that $D$ is ordinary if there exists an ordinary $G_{\mathbb{Q}, S}$-representation $\rho$ such that $D$ is induced by $\rho$.

- While not every pseudorepresentation comes from a representation, we fix this problem by broadening the category of representations to include CayleyHamilton representations. We first define ordinary Cayley-Hamilton representations, and then say a pseudorepresentation is ordinary when there exists an ordinary Cayley-Hamilton representation inducing it.

We fix some notation. We use the letter $\psi$ to denote the functor that associates to a representation its induced pseudorepresentation. Let $\bar{D}=\psi\left(\omega^{-1} \oplus \theta^{-1}\right)$, which is the $\mathbb{F}$-valued residual pseudorepresentation induced by the Galois action on $H$. Write $R_{\bar{D}}$ for the pseudodeformation ring for $\bar{D}$ WWE15, §5.4] with universal object $D_{\bar{D}}^{u}: G_{\mathbb{Q}, S} \rightarrow R_{\bar{D}}$. In this section, $A$ will denote a Noetherian local $W(\mathbb{F})$ algebra with residue field $\mathbb{F}$. If $a \in A$, then $\bar{a} \in \mathbb{F}$ denotes the image of $a$.

3.1. Representations valued in generalized matrix algebras. As in BC09, $\S 1.3]$, we say that a generalized matrix algebra over $A$ is an associative $A$-algebra $E$ equipped with an $A$-algebra isomorphism

$$
E \stackrel{\sim}{\longrightarrow}\left(\begin{array}{ll}
A & B \\
C & A
\end{array}\right)
$$

which we call a GMA structure. That is, we have $E \stackrel{\sim}{\rightarrow} A \oplus B \oplus C \oplus A$ as $A$-modules for some $A$-modules $B$ and $C$, and there is an $A$-linear map $B \otimes_{A} C \rightarrow A$ such that the multiplication in $E$ is given by 2-by-2 matrix multiplication. In this case, $A$ is called the scalar subring of $E$ and $E$ is called an $A$-GMA.

A GMA representation with coefficients in $A$ and residual pseudorepresentation $\bar{D}$ is a homomorphism $\rho: G_{\mathbb{Q}, S} \rightarrow E^{\times}$, such that $E$ is an $A$-GMA, and such that in matrix coordinates, $\rho$ is given by

$$
\sigma \mapsto\left(\begin{array}{cc}
\rho_{1,1}(\sigma) & \rho_{1,2}(\sigma) \\
\rho_{2,1}(\sigma) & \rho_{2,2}(\sigma)
\end{array}\right)
$$

with $\overline{\rho_{1,1}(\sigma)}=\omega^{-1}(\sigma), \overline{\rho_{2,2}(\sigma)}=\theta^{-1}(\sigma)$, and $\overline{\rho_{1,2}(\sigma) \rho_{2,1}(\sigma)}=0$. We emphasize the fact that we fix the order of the diagonal characters.

Given such a $\rho$, there is an induced $A$-valued pseudorepresentation, denoted $\psi_{\mathrm{GMA}}(\rho): G_{\mathbb{Q}, S} \rightarrow A$, given by $\operatorname{tr}(\rho)=\rho_{1,1}+\rho_{2,2}$ and $\operatorname{det}(\rho)=\rho_{1,1} \rho_{2,2}-\rho_{1,2} \rho_{2,1}$; cf. WE15, Prop. 2.23].

3.2. Universality. A Cayley-Hamilton representation with scalar $\operatorname{ring} A$ and residual pseudorepresentation $\bar{D}$ is the data of a pair $\left(\rho: G_{\mathbb{Q}, S} \rightarrow E^{\times}, D: E \rightarrow A\right)$, where $E$ is an associative $A$-algebra such that $D \circ \rho$ is a pseudorepresentation deforming $\bar{D}$. These data must satisfy an additional Cayley-Hamilton condition that, for all $x \in E, x$ must satisfy the characteristic polynomial associated to $x$ by $D$. If $\rho: G_{\mathbb{Q}, S} \rightarrow E^{\times}$is a GMA representation, then $\left(\rho, \psi_{\mathrm{GMA}}(\rho)\right)$ is a Cayley-Hamilton representation.

For our purposes, the important properties of Cayley-Hamilton representations are the following (see [WE15, Prop. 3.6]). 
- There is a universal Cayley-Hamilton representation $\left(\rho^{u}: G_{\mathbb{Q}, S} \rightarrow E_{\bar{D}}^{\times}, D^{u}\right.$ : $E_{\bar{D}} \rightarrow R_{\bar{D}}$ ) with residual pseudorepresentation $\bar{D}$, and the induced pseudorepresentation $D^{u} \circ \rho^{u}$ of $G$ is equal to the universal deformation of $\bar{D}$.

- $E_{\bar{D}}$ is finitely generated as an $R_{\bar{D}}$-module, and $\rho^{u}$ is continuous for the natural adic topology from $R_{\bar{D}}$ on $E_{\bar{D}}$.

- $E_{\bar{D}}$ admits various $R_{\bar{D}}$-GMA structures making $\rho^{u}$ a GMA representation over $R_{\bar{D}}$.

In particular, any Cayley-Hamilton representation with residual pseudorepresentation $\bar{D}$ admits the structure of a GMA representation with residual pseudorepresentation $\bar{D}$. Note that by definition of GMA representation, we have insisted that any such GMA structure satisfies (3.1.1).

Given a GMA structure on $E_{\bar{D}}$, we will write

$$
E_{\bar{D}} \cong\left(\begin{array}{ll}
R_{\bar{D}} & B^{u} \\
C^{u} & R_{\bar{D}}
\end{array}\right)
$$

for the decomposition of $E_{\bar{D}}$ as in (3.1.1) and write $\rho_{i, j}^{u}$ for the corresponding coordinates of $\rho^{u}$. Similarly, for any GMA representation $\rho$ deforming $\bar{D}$, we will write $\rho_{i, j}$ for the induced coordinate decomposition.

3.3. Reducibility. It will be important to understand the notion of a reducible pseudorepresentation and the reducibility ideal in $A$ for an $A$-valued pseudodeformation $D: G_{\mathbb{Q}, S} \rightarrow A$ of $\bar{D}$. We call $D$ reducible if $D=\psi\left(\chi_{1} \oplus \chi_{2}\right)$ for characters $\chi_{i}: G_{\mathbb{Q}, S} \rightarrow A^{\times}$such that $\bar{\chi}_{1}=\omega^{-1}$ and $\bar{\chi}_{2}=\theta^{-1}$. Otherwise, $D$ is called irreducible. Equivalently, $D$ is reducible if $D=\psi_{\mathrm{GMA}}(\rho)$ for some GMA representation $\rho$ with scalar ring $A$ such that $\rho_{1,2}\left(G_{\mathbb{Q}, S}\right) \cdot \rho_{2,1}\left(G_{\mathbb{Q}, S}\right)$ is zero.

Since $\rho_{1,2}^{u}\left(G_{\mathbb{Q}, S}\right)$ and $\rho_{2,1}^{u}\left(G_{\mathbb{Q}, S}\right)$ generate $B^{u}$ and $C^{u}$ respectively as $R_{\bar{D}^{-} \text {-modules, }}$ $D$ is reducible exactly when the image of $B^{u} \otimes_{R_{\bar{D}}} C^{u}$ in $R_{\bar{D}}$ under multiplication vanishes under $R_{\bar{D}} \rightarrow A$. This holds true for any choice of GMA structure. Consequently, we call the image of $B^{u} \otimes_{R_{\bar{D}}} C^{u}$ in $R_{\bar{D}}$ the reducibility ideal of $R_{\bar{D}}$, and its image in $A$ is the reducibility ideal for $D$.

3.4. Ordinary GMA representations. We will say that a representation of $\operatorname{Gal}\left(\overline{\mathbb{Q}}_{p} / \mathbb{Q}_{p}\right)$ on a 2 -dimensional $p$-adic vector space $V$ is ordinary if there exists a 1-dimensional quotient representation $V \rightarrow W$ such that $W(1)$ is unramified. A representation $\rho$ of $G_{\mathbb{Q}, S}$ is ordinary if $\left.\rho\right|_{G_{p}}$ is ordinary. This notion of ordinariness is relatively restrictive compared to other uses of the term, but it will suit our purpose of studying Galois representations associated to ordinary modular forms.

Relative to the ordering of factors in (3.1.1), we have the following definition of an ordinary Cayley-Hamilton representation.

Definition 3.4.1. Let $\rho: G_{\mathbb{Q}, S} \rightarrow E^{\times}$be a Cayley-Hamilton representation with scalar ring $A$ and induced pseudorepresentation $\bar{D}$. We call $\rho$ ordinary provided that it admits a GMA structure such that

(1) $\rho_{1,2}\left(G_{p}\right)=0$, and

(2) $\left.\rho_{1,1}\right|_{I_{p}} \simeq \kappa_{\mathrm{cyc}}^{-1} \otimes_{\mathbb{Z}_{p}} A$,

where $\kappa_{\text {cyc }}$ is the $p$-adic cyclotomic character.

Remark 3.4.2. The condition that $\omega$ and $\theta$ are locally $p$-distinguished, i.e., $\left.\omega\right|_{G_{p}} \neq$ $\left.\theta\right|_{G_{p}}$, is critically necessary to making this definition sensible. This condition is equivalent to the assumption (b) of 2.1 
Example 3.4.3. Let $\rho: G_{\mathbb{Q}, S} \rightarrow \operatorname{Aut}_{A}(V)$ be a representation on a free rank 2 $A$-module $V$ with induced pseudorepresentation $\bar{D}$. This amounts to a CayleyHamilton representation over $A$ valued in $\operatorname{End}_{A}(V)$. We claim that $\rho$ is ordinary according to Definition 3.4.1 if and only if there is a quotient character $\alpha$ of $\left.V\right|_{G_{p}}$ such that $\bar{\alpha}=\omega^{-1}$ and $\left.\alpha\right|_{I_{p}}=\left.\left(\kappa_{\text {cyc }}^{-1} \otimes_{\mathbb{Z}_{p}} A\right)\right|_{I_{p}}$. Indeed, the latter condition is true if and only if there exists a basis $V \cong A^{2}$ such that $\rho: G_{\mathbb{Q}, S} \rightarrow \mathrm{GL}_{2}(A)$ satisfies Definition 3.4.1. Observe that a choice of basis for $V$ induces a GMA structure $\operatorname{End}_{A}(V) \stackrel{\sim}{\rightarrow} M_{2}(A)$ on $\operatorname{End}_{A}(V)$.

This definition is slightly more restrictive than the definition of "ordinary representation" given by some authors. Nonetheless, our definition can be useful for studying those more general representations (see \$5.2).

Example 3.4.4. The motivating example of an ordinary GMA representation is the $\mathfrak{h}\left[G_{\mathbb{Q}, S}\right]$-module $H$ given by the cohomology of modular curves. There exist isomorphisms of $\mathfrak{h}$-modules $H \stackrel{\sim}{\rightarrow} \mathfrak{h} \oplus \mathfrak{h}^{\vee}$, where $\mathfrak{h}^{\vee}$ is the dualizing module of $\mathfrak{h}$ (see [WWE15, §3.4]). Because $\operatorname{End}_{\mathfrak{h}}\left(\mathfrak{h}^{\vee}\right) \cong \mathfrak{h}$, any such isomorphism determines a GMA representation $\rho_{H}: G_{\mathbb{Q}, S} \rightarrow \operatorname{Aut}_{\mathfrak{h}}(H)$; moreover, there exists a choice of isomorphism such that

$$
G_{\mathbb{Q}, S} \longrightarrow \operatorname{End}_{\mathfrak{h}}(H) \stackrel{\sim}{\longrightarrow}\left(\begin{array}{cc}
\operatorname{End}_{\mathfrak{h}}(\mathfrak{h}) & \operatorname{Hom}_{\mathfrak{h}}\left(\mathfrak{h}, \mathfrak{h}^{\vee}\right) \\
\operatorname{Hom}_{\mathfrak{h}}\left(\mathfrak{h}^{\vee}, \mathfrak{h}\right) & \operatorname{End}_{\mathfrak{h}}\left(\mathfrak{h}^{\vee}\right)
\end{array}\right) \cong\left(\begin{array}{cc}
\mathfrak{h} & \mathfrak{h}^{\vee} \\
\operatorname{Hom}_{\mathfrak{h}}\left(\mathfrak{h}^{\vee}, \mathfrak{h}\right) & \mathfrak{h}
\end{array}\right)
$$

satisfies conditions (1) and (2) of Definition 3.4.1 relative to the resulting GMA structure.

Here is a summary of the results of WWE15 on ordinary Cayley-Hamilton representations.

\section{Proposition 3.4.5.}

(1) There is a universal ordinary Cayley-Hamilton algebra $E_{\bar{D}}^{\text {ord }}$, a quotient of $E_{\bar{D}}$, such that a Cayley-Hamilton representation $G_{\mathbb{Q}, S} \rightarrow E^{\times}$with residual pseudorepresentation $\bar{D}$ is ordinary if and only if its map $E_{\bar{D}} \rightarrow E$ factors through $E_{\bar{D}}^{\text {ord }}$.

(2) There is a universal reducible ordinary Cayley-Hamilton algebra $E_{\bar{D}}^{\mathrm{red}}$, a quotient of $E_{\bar{D}}$, such that a Cayley-Hamilton representation $G_{\mathbb{Q}, S} \rightarrow E^{\times}$ with residual pseudorepresentation $\bar{D}$ is reducible ordinary if and only if its map $E_{\bar{D}} \rightarrow E$ factors through $E_{\bar{D}}^{\mathrm{red}}$.

(3) $\rho_{H}: G_{\mathbb{Q}, S} \rightarrow \operatorname{Aut}_{\mathfrak{h}}(H)$ is an ordinary Cayley-Hamilton representation.

Proof. Statement (1) comes from [WWE15, Proposition 5.9.7, (2) comes from Proposition 7.3.1, and (3) comes from Theorem 7.1.2.

For the rest of the paper, we fix a GMA structure on $E_{\bar{D}}^{\text {ord }}$ such that (1) and (2) of Definition 3.4.1 are satisfied. By its universal property (part (1) of Proposition 3.4.5), this induces a GMA structure on all ordinary Cayley-Hamilton representations. We refer to an ordinary Cayley-Hamilton representation with this choice of GMA structure as an ordinary GMA representation.

3.5. Ordinary pseudorepresentations. Having established a notion of ordinary GMA representation, we can now define ordinary pseudorepresentations. 
Definition 3.5.1. Let $D: G_{\mathbb{Q}, S} \rightarrow A$ be a pseudorepresentation deforming $\bar{D}$. Then we call $D$ ordinary if there exists an ordinary GMA representation $\rho: G_{\mathbb{Q}, S} \rightarrow$ $E^{\times}$with scalar ring $A$ such that $D=\psi_{\text {GMA }}(\rho)$.

We write $R_{\bar{D}}^{\text {ord }}$ for the scalar ring of $E_{\bar{D}}^{\text {ord }}$, which admits a pseudorepresentation $D_{\bar{D}}^{\text {ord }}: G_{\mathbb{Q}, S} \rightarrow R_{\bar{D}}^{\text {ord }}$ defined as the composition of $D_{\bar{D}}^{u}: G_{\mathbb{Q}, S} \rightarrow R_{\bar{D}}$ with $R_{\bar{D}} \rightarrow$ $R_{\bar{D}}^{\text {ord }}$. We have shown in [WWE15, Thm. 5.10.4] that the ring $R_{\bar{D}}^{\text {ord }}$ represents the functor of ordinary pseudodeformations of $\bar{D}$, with universal object $D_{\bar{D}}^{\text {ord }}$. We write $\mathcal{J} \subset R_{\bar{D}}^{\text {ord }}$ for the reducibility ideal of $D_{\bar{D}}^{\text {ord }}$.

Remark 3.5.2. The reason for introducing GMA representations is to make Definition 3.5 .1 not every pseudodeformation of $\bar{D}$ comes from a representation, but every pseudodeformation comes from a GMA representation.

By definition, we see that the modular pseudorepresentation $\psi_{\mathrm{GMA}}\left(\rho_{H}\right): G_{\mathbb{Q}, S} \rightarrow$ $\mathfrak{h}$ arising from $\rho_{H}$ (as defined in Example 3.4.4) is ordinary. It can be extended to an $\mathfrak{H}$-valued pseudorepresentation with the following properties.

Proposition 3.5.3. There is a pseudorepresentation $D_{H}: G_{\mathbb{Q}, S} \rightarrow \mathfrak{H}$ that is ordinary, deforms $\bar{D}$, and satisfies $D_{H} \otimes_{\mathfrak{H}} \mathfrak{h}=\psi_{\mathrm{GMA}}\left(\rho_{H}\right)$. The corresponding map $\phi: R_{\bar{D}}^{\text {ord }} \rightarrow \mathfrak{H}$ is:

(1) a map of augmented $\Lambda$-algebras, where the augmentation ideals are the reducibility ideals $\mathcal{J} \subset R_{\bar{D}}^{\text {ord }}$ of $D_{\bar{D}}^{\text {ord }}$ and $\mathcal{I} \subset \mathfrak{H}$ of $D_{H}$, and

(2) surjective.

Proof. The pseudorepresentation $D_{H}$ is constructed by gluing $\psi_{\mathrm{GMA}}\left(\rho_{H}\right)$ together with the Eisenstein pseudorepresentation, and it follows that $D_{H} \otimes_{\mathfrak{H}} \mathfrak{h}=\psi_{\text {GMA }}\left(\rho_{H}\right)$ and that the reducibility ideal is $\mathcal{I} \subset \mathfrak{H}$ WWE15, Cor. 7.1.3].

Then (2) follows from [WWE15, Lem. 7.1.4], and (1) follows from the fact that $R_{\bar{D}}^{\text {ord }} / \mathcal{J} \cong \Lambda$ WWE15, Prop. 7.3.1].

Consequently, the functor of reducible ordinary pseudorepresentations is represented by $\Lambda$, and $\Lambda$ is the scalar ring of $E_{\bar{D}}^{\text {red }}$.

\section{NeW RESUlts}

With the overview of [WWE15] complete, we prove the main theorems.

4.1. Reducible representations and class groups. Let us write $E_{\bar{D}}^{\text {ord }}$ and $E_{\bar{D}}^{\text {red }}$ in GMA form as

$$
E_{\bar{D}}^{\mathrm{ord}} \cong\left(\begin{array}{ll}
R_{\bar{D}}^{\mathrm{ord}} & B^{\mathrm{ord}} \\
C^{\text {ord }} & R_{\bar{D}}^{\text {ord }}
\end{array}\right), \quad E_{\bar{D}}^{\mathrm{red}} \cong\left(\begin{array}{cc}
\Lambda & B^{\text {red }} \\
C^{\text {red }} & \Lambda
\end{array}\right)
$$

Our goal is to control $\mathcal{J} / \mathcal{J}^{2}$ using Galois cohomology. For this, we use the surjection $B^{\text {ord }} \otimes_{R_{\bar{D}}^{\text {ord }}} C^{\text {ord }} \rightarrow \mathcal{J}$ discussed in $\$ 3.3$. We also use the fact that the map $E_{\bar{D}}^{\text {red }} \rightarrow E_{\bar{D}}^{\text {ord }} / \mathcal{J} E_{\bar{D}}^{\text {ord }}$, which exists because the target receives a reducible ordinary GMA representation, is surjective [WWE15, Prop. 7.3.1(4)]. (Actually, it is an isomorphism, but that will not be used here.) Composing these surjections, we have

$$
B^{\text {red }} \otimes_{\Lambda} C^{\text {red }} \rightarrow B^{\text {ord }} / \mathcal{J} \otimes_{\Lambda} C^{\text {ord }} / \mathcal{J} \rightarrow \mathcal{J} / \mathcal{J}^{2}
$$

(cf. [WWE15, Prop. 5.7.2]). The main result is the following. 
Proposition 4.1.2. $E_{\bar{D}}^{\mathrm{red}}$ is determined as follows.

(1) There exists a natural isomorphism

$$
X_{\chi}(1) \stackrel{\sim}{\longrightarrow} B^{\text {red }} .
$$

(2) Assume that $X_{\theta}=0$. Then there exists a natural isomorphism

$$
\mathfrak{X}_{\chi^{-1}}^{\#}(1) \stackrel{\sim}{\longrightarrow} C^{\text {red }} \text {. }
$$

Moreover, $C^{\text {red }}$ is free of rank 1 over $\Lambda$.

First, some lemmas. We will need the following notation, which is particular to 4.1. We will abbreviate $H^{i}(\mathbb{Z}[1 / N p],-)$ and $H_{(c)}^{i}(\mathbb{Z}[1 / N p],-)$ to $H^{i}(-)$ and $H_{(c)}^{i}(-)$, respectively. For $n \in \mathbb{Z}_{\geq 1}$, we will let $H_{n}^{i}(-)$ denote $\bigoplus_{\ell \mid n} H^{i}\left(\mathbb{Q}_{\ell},-\right)$, where $\ell$ runs over prime divisors of $n$. Likewise, for a $\Lambda$-module $M$ (with trivial $G_{\mathbb{Q}, S}$-action), we will write $M^{\#}$ for $\Lambda^{\#} \otimes_{\Lambda} M$ and write $M^{\langle-\rangle}$for $\Lambda^{\langle-\rangle} \otimes_{\Lambda} M$.

Lemma 4.1.3. For any finitely generated $\Lambda$-module $M$ and any $\ell \mid N$ we have $H_{\ell}^{1}\left(M^{\langle-\rangle}(-1)\right)=0$. In particular, $H_{N p}^{1}\left(M^{\langle-\rangle}(-1)\right)=H_{p}^{1}\left(M^{\langle-\rangle}(-1)\right)$.

Proof. Let $\bar{M}=M / \mathfrak{m}_{\Lambda} M$, where $\mathfrak{m}_{\Lambda}$ is the maximal ideal of $\Lambda$. By Nakayama's lemma, it suffices to show that $H_{\ell}^{1}\left(\bar{M}^{\langle-\rangle}(-1)\right)=0$. By the Euler characteristic formula and local Tate duality, we have

$$
\operatorname{dim}\left(H_{\ell}^{1}\left(\bar{M}^{\langle-\rangle}(-1)\right)\right)=\operatorname{dim}\left(H_{\ell}^{0}\left(\bar{M}^{\langle-\rangle}(-1)\right)\right)+\operatorname{dim}\left(H_{\ell}^{0}\left(\bar{M}^{\#}(2)\right)\right) .
$$

The Galois action on $\bar{M}^{\langle-\rangle}(-1)$ and $\bar{M}^{\#}(2)$ is via the characters $\theta \omega^{-1}$ and $\theta^{-1} \omega^{2}$, respectively. Both these characters are non-trivial at $\ell$ because $\omega$ is trival at $\ell$ and we assume that $\theta$ is primitive. This implies that the $H_{\ell}^{0}$ groups appearing in (4.1.4) are 0 , so $H_{\ell}^{1}\left(\bar{M}^{\langle-\rangle}(-1)\right)=0$.

Lemma 4.1.5. Functorially in finitely generated $\Lambda$-modules $M$, we have isomorphisms

$$
\operatorname{Hom}_{\Lambda}\left(B^{\mathrm{red}}, M\right) \stackrel{\sim}{\longrightarrow} H_{(c)}^{1}\left(M^{\langle-\rangle}(-1)\right)
$$

and

$$
\operatorname{Hom}_{\Lambda}\left(C^{\mathrm{red}}, M\right) \stackrel{\sim}{\longrightarrow} H^{1}\left(M^{\#}(1)\right) .
$$

Proof. For [4.1.7), BC09, Thm. 1.5.5] tells us that there is a natural $\Lambda$-linear injective map $i_{C}: \operatorname{Hom}_{\Lambda}\left(C^{\text {red }}, M\right) \hookrightarrow H^{1}\left(M^{\#}(1)\right)$ when $M$ is a cyclic module. But nothing about the proof depends upon $M$ being cyclic, so we have injectivity in general.

An element of $H^{1}\left(M^{\#}(1)\right)$ results in a short exact sequence of $\Lambda\left[G_{\mathbb{Q}, S}\right]$-modules $0 \rightarrow M^{\#} \rightarrow \mathcal{E} \rightarrow \Lambda(-1) \rightarrow 0$. Choose an element $x \in \mathcal{E}$ mapping to $1 \in \Lambda$ and write $f: G_{\mathbb{Q}, S} \rightarrow M$ for the map $\gamma \mapsto \gamma \cdot x-\kappa_{\text {cyc }}^{-1}(\gamma) x$. Then we have a GMA representation

$$
\rho: G_{\mathbb{Q}, S} \longrightarrow\left(\begin{array}{cc}
\Lambda & 0 \\
M & \Lambda
\end{array}\right), \quad \rho(\gamma)=\left(\begin{array}{cc}
\kappa_{\mathrm{cyc}}^{-1}(\gamma) & 0 \\
f(\gamma) & \langle\gamma\rangle^{-1}
\end{array}\right)
$$

By the universal property of $E_{\bar{D}}^{\text {red }}$, there exists a unique map $C^{\text {red }} \rightarrow M$ induced by this representation. This construction is an inverse to the construction of $i_{C}$ in [BC09, Thm. 1.5.5], so we have proved that (4.1.7) is an isomorphism.

Recall the definition of $H_{(c)}^{1}$ as the cohomology of a mapping cone. Note that $H_{N p}^{0}\left(M^{\langle-\rangle}(-1)\right)=0$ for any $M$. This follows from the fact that the residual 
character $\chi$ is non-trivial on decomposition groups at all primes dividing $N p$. Consequently, $H_{(c)}^{1}\left(M^{\langle-\rangle}(-1)\right)$ is naturally isomorphic to the subset of $H^{1}\left(M^{\langle-\rangle}(-1)\right)$ whose restriction to $H_{N p}^{1}\left(M^{\langle-\rangle}(-1)\right)$ is zero. By Lemma 4.1.3, $H_{N p}^{1}\left(M^{\langle-\rangle}(-1)\right)=$ $H_{p}^{1}\left(M^{\langle-\rangle}(-1)\right)$.

We have an injection

$$
i_{B}: \operatorname{Hom}_{\Lambda}\left(B^{\mathrm{red}}, M\right) \hookrightarrow H_{(c)}^{1}\left(M^{\langle-\rangle}(-1)\right) \subset H^{1}\left(M^{\langle-\rangle}(-1)\right)
$$

like $i_{C}$ above, because any extension of $\Lambda^{\langle-\rangle}$by $M(-1)$ realized by a GMA map

$$
E_{\bar{D}}^{\mathrm{red}} \rightarrow\left(\begin{array}{cc}
\Lambda & M \\
0 & \Lambda
\end{array}\right)
$$

induces a trivial extension of $G_{p}$-representations. The same argument as above shows that $i_{B}$ is surjective.

Now, we prove Proposition 4.1.2

Proof. We will use the Yoneda lemma for finitely generated $\Lambda$-modules to determine $B^{\text {red }}$ and $C^{\text {red }}$. Indeed, $B^{\text {red }}$ and $C^{\text {red }}$ are finitely generated by the fact that $E_{\bar{D}}$ is a finitely generated $R_{\bar{D}}$-module (see $\$ 3.2$ ) and the construction of $E_{\bar{D}}^{\text {red }}$.

Let's begin with $B^{\text {red }}$. Let $M$ be an arbitrary finitely generated $\Lambda$-module. We will use the spectral sequence of Proposition 2.2.1.

$$
\operatorname{Ext}_{\Lambda}^{p}\left(H^{3-q}\left(\Lambda^{\#}(2)\right), M\right) \Longrightarrow H_{(c)}^{p+q}\left(M^{\langle-\rangle}(-1)\right) .
$$

From [WWE15, Cor. 6.3.1], we have

$$
H^{0}\left(\Lambda^{\#}(2)\right) \cong 0, \quad H^{1}\left(\Lambda^{\#}(2)\right) \cong 0, \quad H^{2}\left(\Lambda^{\#}(2)\right) \cong X_{\chi}(1) .
$$

The spectral sequence degenerates to yield a functorial isomorphism

$$
\operatorname{Hom}_{\Lambda}\left(X_{\chi}(1), M\right) \stackrel{\sim}{\longrightarrow} H_{(c)}^{1}\left(M^{\langle-\rangle}(-1)\right) .
$$

From this and (4.1.6), we see that $X_{\chi}(1)$ and $B^{\text {red }}$ represent the same functor $M \mapsto H_{(c)}^{1}\left(M^{\langle-\rangle}(-1)\right)$. Then the Yoneda lemma implies that $X_{\chi}(1) \stackrel{\sim}{\longrightarrow} B^{\text {red }}$.

We now similarly calculate $C^{\text {red }}$. Let $M$ be any finitely generated $\Lambda$-module, and consider the spectral sequence

$$
\operatorname{Ext}_{\Lambda}^{p}\left(H_{(c)}^{3-q}\left(\Lambda^{\langle-\rangle}\right), M\right) \Longrightarrow H^{p+q}\left(M^{\#}(1)\right)
$$

As in the proof of Proposition 2.2.6, we have $H_{(c)}^{2}\left(\Lambda^{\langle-\rangle}\right) \cong \mathfrak{X}_{\chi^{-1}}^{\#}(1)$. Similar arguments show that $H_{(c)}^{3}\left(\Lambda^{\langle-\rangle}\right)=0$ and, using the weak Leopoldt conjecture (see [NSW08, Thm. 10.3.22]), that $H_{(c)}^{1}\left(\Lambda^{\langle-\rangle}\right)=0$. Then the spectral sequence degenerates to yield $\operatorname{Hom}_{\Lambda}\left(\mathfrak{X}_{\chi^{-1}}^{\#}(1), M\right) \cong H^{1}\left(M^{\#}(1)\right)$. As above, using (4.1.7) and the Yoneda lemma, we obtain $\mathfrak{X}_{\chi^{-1}}^{\#}(1) \stackrel{\sim}{\rightarrow} C^{\text {red }}$.

4.2. A version of Wiles's numerical criterion. Having controlled $\mathcal{J} / \mathcal{J}^{2}$ in terms of Iwasawa class groups, we will now make use of a version of Wiles's numerical criterion [Wil95, Appendix] to prove our $R=T$ theorem. We thank Eric Urban for suggesting that the numerical criterion might be used to improve an earlier version. We follow the exposition of dSRS97. 
Consider the diagram

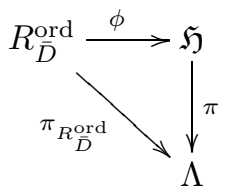

where $\phi$ arises from Proposition 3.5.3. In this situation, de Smit, Rubin, and Schoof prove the following theorem on the way to giving a proof of Wiles's criterion.

Theorem 4.2.1 (dSRS97, Thm. of $\S 3$ (p. 9)]). The map $\phi$ is an isomorphism of complete intersections if and only if $\phi\left(\operatorname{Fitt}_{R_{\bar{D}}^{\text {ord }}}(\mathcal{J})\right) \not \subset \mathfrak{m}_{\Lambda} \mathfrak{H}$.

We will use this theorem to show that $\phi$ is an isomorphism under the assumption that $X_{\theta}=0$. The proof is inspired by the proof of Criteria I in dSRS97. We first prove some preliminary results. The notation 'Fitt' that appears in the theorem refers to Fitting ideals, which are reviewed in dSRS97, §1]. We will make frequent use of the following well-known properties of Fitting ideals (see dSRS97, Prop. $1.1])$.

Lemma 4.2.2. Let $A$ be a ring, let $M$ be a finitely presented $A$-module, and let $B$ be an A-algebra. Then:

(1) $\operatorname{Fitt}_{B}\left(M \otimes_{A} B\right)=\operatorname{Fitt}_{A}(M) \cdot B$.

(2) $\operatorname{Fitt}_{A}(M) \subset \operatorname{Ann}_{A}(M)$.

Before stating the next lemma, we summarize some known results about the Eisenstein ideals $\mathcal{I}$ and $I$ (see [WWE15, Prop. 3.2.5 and Lem. 3.2.9]): the natural map $\mathcal{I} \rightarrow I$ is an isomorphism, $\mathfrak{h} / I \cong \Lambda / \xi$, and $\operatorname{Ann}_{\mathfrak{H}}(\mathcal{I})=\operatorname{ker}(\mathfrak{H} \rightarrow \mathfrak{h})$. In particular, $I$ is a faithful $\mathfrak{h}$-module.

Lemma 4.2.3. We have $\pi\left(\operatorname{Fitt}_{\mathfrak{H}}(\mathcal{I})\right)=\operatorname{Fitt}_{\Lambda}\left(I / I^{2}\right) \subset(\xi)$ as ideals of $\Lambda$.

Proof. By Lemma 4.2.2(1), we have

$$
\pi\left(\operatorname{Fitt}_{\mathfrak{H}}(\mathcal{I})\right)=\operatorname{Fitt}_{\Lambda}\left(\mathcal{I} \otimes_{\mathfrak{H}} \Lambda\right) .
$$

Since $\mathcal{I} \otimes_{\mathfrak{H}} \Lambda=\mathcal{I} / \mathcal{I}^{2} \cong I / I^{2}$, we have $\pi\left(\operatorname{Fitt}_{\mathfrak{H}}(\mathcal{I})\right)=\operatorname{Fitt}_{\Lambda}\left(I / I^{2}\right)$.

Since $I$ is a faithful $\mathfrak{h}$-module, Lemma 4.2.2(2) implies that $\operatorname{Fitt}_{\mathfrak{h}}(I)=0$. Applying Lemma 4.2.2(1), we have $\operatorname{Fitt}_{\mathfrak{h} / I}\left(I / I^{2}\right)=0$. Recalling that $\mathfrak{h} / I \cong \Lambda / \xi$, another application of the same lemma gives $\operatorname{Fitt}_{\Lambda}\left(I / I^{2}\right) \subset(\xi)$.

Proposition 4.2.4. Assume that $X_{\theta}=0$. Then the $\Lambda$-modules $X_{\chi}(1), \mathcal{J} / \mathcal{J}^{2}$, $\mathcal{I} / \mathcal{I}^{2}$, and $I / I^{2}$ are all isomorphic and they all have Fitting ideal over $\Lambda$ equal to $(\xi)$.

Remark 4.2.5. Sharifi has studied a map from class groups to $I / I^{2}$ similar to the one that appears in the following proof; see the map of [Sha07, Thm. 5.2].

Proof. From (4.1.1), we have the surjections

$$
B^{\text {red }} \otimes_{\Lambda} C^{\text {red }} \rightarrow \mathcal{J} / \mathcal{J}^{2} \rightarrow \mathcal{I} / \mathcal{I}^{2} \stackrel{\sim}{\rightarrow} I / I^{2} .
$$

By Proposition 4.1.2, we have $B^{\text {red }} \cong X_{\chi}(1)$ and $C^{\text {red }} \cong \mathfrak{X}_{\chi^{-1}}^{\#}(1)$, and so by Proposition 2.2.6 we have $C^{\text {red }} \simeq \Lambda$. Hence we have a surjection

$$
\Theta: X_{\chi}(1) \rightarrow I / I^{2} \text {. }
$$


But by the previous lemma, $\operatorname{Fitt}_{\Lambda}\left(I / I^{2}\right) \subset(\xi)=\operatorname{char}_{\Lambda}\left(X_{\chi}(1)\right)$. This implies that the $\operatorname{ker}(\Theta)$ is finite (see Wak15a, Lem. A.7] for example), and hence $\operatorname{ker}(\Theta)=0$, since $X_{\chi}(1)$ has no finite submodule by Ferrero-Washington [FW79]. Thus $\Theta$ is an isomorphism, and therefore so are all of the maps in (4.2.6).

Since the modules are all isomorphic, it suffices to compute $\operatorname{Fitt}_{\Lambda}\left(X_{\chi}(1)\right)$, which is well-known to be $(\xi)$. Indeed, by Ferrero-Washington and Lemma 2.2.5, $X_{\chi}(1)$ has projective dimension 1 . Therefore the ideal $\operatorname{Fitt}_{\Lambda}\left(X_{\chi}(1)\right)$ is principal. This $\operatorname{implies}_{F^{\prime}} \operatorname{itt}_{\Lambda}\left(X_{\chi}(1)\right)=\operatorname{char}_{\Lambda}\left(X_{\chi}(1)\right)$ (see Wak15a, Lem. A.6], for example) which is $(\xi)$ by definition.

Lemma 4.2.7. We have $\operatorname{Ann}_{\mathfrak{H}}(\mathcal{I})=\operatorname{ker}(\mathfrak{H} \rightarrow \mathfrak{h})$ and, in particular, $\operatorname{Ann}_{\mathfrak{H}}(\mathcal{I}) \not \subset$ $\mathfrak{m}_{\Lambda} \mathfrak{H}$. The restriction of $\pi$ to $\operatorname{Ann}_{\mathfrak{H}}(\mathcal{I})$ induces an isomorphism

$$
\left.\pi\right|_{\text {Ann }}: \operatorname{Ann}_{\mathfrak{H}}(\mathcal{I}) \stackrel{\sim}{\longrightarrow}(\xi) .
$$

Proof. That $\operatorname{Ann}_{\mathfrak{H}}(\mathcal{I})=\operatorname{ker}(\mathfrak{H} \rightarrow \mathfrak{h})$ and the fact that $\left.\pi\right|_{\text {Ann }}$ is an isomorphism follows from [WWE15, Prop. 3.2.5]. To see that $\operatorname{Ann}_{\mathfrak{H}}(\mathcal{I}) \not \subset \mathfrak{m}_{\Lambda} \mathfrak{H}$, note that $\mathfrak{H} \rightarrow \mathfrak{h}$ is a surjection of free $\Lambda$-modules of distinct rank, and so $\operatorname{Ann}_{\mathfrak{H}}(\mathcal{I})=\operatorname{ker}(\mathfrak{H} \rightarrow \mathfrak{h})$ is a non-zero $\Lambda$-free direct summand of $\mathfrak{H}$.

Theorem 4.2.8. Assume that $X_{\theta}=0$. Then $\phi: R_{\bar{D}}^{\text {ord }} \rightarrow \mathfrak{H}$ is an isomorphism of complete intersections.

Proof. By Theorem 4.2.1 it suffices to show that

$$
\phi\left(\operatorname{Fitt}_{R_{\bar{D}}^{\text {ord }}}(\mathcal{J})\right) \not \subset \mathfrak{m}_{\Lambda} \mathfrak{H} .
$$

By Lemma 4.2.2(1) and Proposition 4.2.4, we have

$$
\pi_{R_{\bar{D}}^{\text {ord }}}\left(\operatorname{Fitt}_{R_{\bar{D}}^{\text {ord }}}(\mathcal{J})\right)=\operatorname{Fitt}_{\Lambda}\left(\mathcal{J} \otimes_{R_{\bar{D}}^{\text {ord }}} \Lambda\right)=\operatorname{Fitt}_{\Lambda}\left(\mathcal{J} / \mathcal{J}^{2}\right)=(\xi) .
$$

On the other hand, Lemma 4.2.2 $(2)$ implies that $\operatorname{Fitt}_{R_{\bar{D}}^{\text {ord }}}(\mathcal{J}) \subset \operatorname{Ann}_{R_{\bar{D}}^{\text {ord }}}(\mathcal{J})$, and, since $\left.\phi\right|_{\mathcal{J}}: \mathcal{J} \rightarrow \mathcal{I}$ is surjective, we have $\phi\left(\operatorname{Ann}_{R_{\bar{D}}^{\text {ord }}}(\mathcal{J})\right) \subset \operatorname{Ann}_{\mathfrak{H}}(\mathcal{I})$. This implies that $\phi\left(\operatorname{Fitt}_{R_{\bar{D}}^{\text {ord }}}(\mathcal{J})\right) \subset \operatorname{Ann}_{\mathfrak{H}}(\mathcal{I})$. Now we have

$$
\pi_{R_{\bar{D}}^{\text {ord }}}\left(\operatorname{Fitt}_{R_{\bar{D}}^{\text {ord }}}(\mathcal{J})\right)=\pi\left(\phi\left(\operatorname{Fitt}_{R_{\bar{D}}^{\text {ord }}}(\mathcal{J})\right)\right)=\left.\pi\right|_{\operatorname{Ann}}\left(\phi\left(\operatorname{Fitt}_{R_{\bar{D}}^{\text {ord }}}(\mathcal{J})\right)\right) .
$$

But we know that $\pi_{R_{\bar{D}}^{\text {ord }}}\left(\operatorname{Fitt}_{R_{\bar{D}}^{\text {ord }}}(\mathcal{J})\right)=(\xi)$, and $\pi\left(\operatorname{Ann}_{\mathfrak{H}}(\mathcal{I})\right)=(\xi)$, so we have

$$
\left.\pi\right|_{\operatorname{Ann}}\left(\phi\left(\operatorname{Fitt}_{R_{\bar{D}}^{\text {ord }}}(\mathcal{J})\right)\right)=\left.\pi\right|_{\operatorname{Ann}_{1}\left(\operatorname{Ann}_{\mathfrak{H}}(\mathcal{I})\right)} .
$$

Since $\left.\pi\right|_{\text {Ann }}$ is an isomorphism by Lemma 4.2.7, we conclude that $\phi\left(\operatorname{Fitt}_{R_{\bar{D}}^{\text {ord }}}(\mathcal{J})\right)=$ $\operatorname{Ann}_{\mathfrak{H}}(\mathcal{I})$. It then follows from Lemma 4.2.7 that $\phi\left(\operatorname{Fitt}_{R_{\bar{D}}^{\text {ord }}}(\mathcal{J})\right) \not \subset \mathfrak{m}_{\Lambda} \mathfrak{H}$.

Remark 4.2.9. This answers in the affirmative a question of Sharifi [Sha09, §5] whether $\mathfrak{H}$ is Gorenstein when $X_{\theta}=0$. As noted in [Sha09, it follows from work of Ohta that $\mathfrak{H}$ is Gorenstein when $\mathfrak{X}_{\theta}=0$, and so our result improves on Ohta's. It was proven in Wak15b, Thm. 1.2] that $\mathfrak{H}$ is Gorenstein when $X_{\theta}=0$ under the additional assumption of Sharifi's conjecture. 


\section{Applications}

5.1. Toward Sharifi's conjecture. We have the following immediate corollary.

Corollary 5.1.1. Assume that $X_{\theta}=0$ and that $X_{\chi}(1)$ is cyclic. Then the ideals $\mathcal{J} \subset R_{\bar{D}}^{\text {ord }}, \mathcal{I} \subset \mathfrak{H}$, and $I \subset \mathfrak{h}$ are all principal, and both $\mathfrak{H}$ and $\mathfrak{h}$ are complete intersections.

Proof. By Proposition 4.2.4 we have $X_{\chi}(1) \simeq \mathcal{J} / \mathcal{J}^{2} \cong \mathcal{I} / \mathcal{I}^{2} \cong I / I^{2}$, and so if $X_{\chi}(1)$ is cyclic, then each ideal is principal by Nakayama's lemma. Since $I$ is faithful as an $\mathfrak{h}$-module, it must be generated by a non-zero divisor. Then since $\mathfrak{h} / I \cong \Lambda / \xi$ is a complete intersection and $I$ is generated by a regular sequence, $\mathfrak{h}$ is a complete intersection (see BH93, Thm. 2.3.4(a), p. 75], for example). Theorem 4.2 .8 implies that $\mathfrak{H}$ is a complete intersection.

These results have applications to Sharifi's conjecture. Recall that Sharifi's conjecture states that two maps $\Upsilon$ and $\varpi$ are isomorphisms [Sha11. In [FK12, FKS14] this conjecture was refined to state that $\Upsilon$ and $\varpi$ are mutually inverse. (See also WWE15, §8.1] for a review of Sharifi's conjecture using the same notation as this paper.)

Corollary 5.1.2. Consider the maps

$$
\Upsilon: X_{\chi}(1) \rightarrow H^{-} / I H^{-} \text {and } \varpi: H^{-} / I H^{-} \rightarrow X_{\chi}(1)
$$

defined by Sharifi. If $X_{\theta}=0$, then $\Upsilon$ is an isomorphism. If, in addition, $X_{\chi}(1)$ is cyclic, then $\varpi$ is an isomorphism as well. Finally, if, in addition, $\xi_{\chi}$ has no multiple root, then they are mutual inverses.

Proof. By Theorem 4.2.8, if $X_{\theta}=0$, then $\mathfrak{H}$ is Gorenstein. It is known that if $\mathfrak{H}$ is Gorenstein, then $\Upsilon$ is an isomorphism (see [Sha11, Prop. 4.10]). If $X_{\chi}(1)$ is cyclic, then the previous corollary implies that $\mathfrak{H}$ and $\mathfrak{h}$ are complete intersections, and hence Gorenstein. The result now follows by work of Fukaya-Kato.

Indeed, since $\mathfrak{h}$ is Gorenstein and $H^{-}$is a dualizing module over $\mathfrak{h}$ (see WWE15, Cor. 3.4.2]), we see that $H^{-} / I H^{-} \simeq \mathfrak{h} / I \cong \Lambda / \xi$, which has no $p$-torsion. Moreover, the fact that $\mathfrak{h}$ is Gorenstein implies the condition $C(\mathfrak{h})$ of Fukaya-Kato by FK12, $\S 7.2 .10]$. Then the final two claims follow from Theorems 7.2.8 and 7.2.7 of [FK12], respectively.

5.2. Residually reducible modularity. In this subsection, we deduce from the main Theorem 4.2.8 a new proof of a known modularity result for ordinary Galois representations. This may be viewed as a verification that our notion of ordinary GMA representation in 93.4 can be usefully applied to ordinary Galois representations as they are usually known. We emphasize that from now on we use the word "ordinary" exclusively to refer to Definition 3.4.1

Remark 5.2.1. Unlike the rest of this paper, we are not assuming the running assumptions about $\theta, p$, and $N$ from $\$ 2.1$ in this subsection. Rather, we will verify that the running assumptions follow from the assumptions of Theorem 5.2.4.

One of the main results of Skinner and Wiles's work [SW99] is

Theorem 5.2.2 (Skinner-Wiles). Suppose that $\rho: G_{\mathbb{Q}} \rightarrow \operatorname{Aut}_{F}(V)$ is continuous, irreducible, and ramified at finitely many primes, where $V$ is a 2-dimensional 
$F$-vector space, $F / \mathbb{Q}_{p}$ is a finite extension, and $p$ is odd. Suppose that $\bar{\rho}^{\text {ss } \simeq}$ $\omega^{-1} \oplus \theta^{-1}$ and that

(1) $\left.\theta\right|_{G_{p}} \neq\left.\omega\right|_{G_{p}}$,

(2) $\left.\rho\right|_{I_{p}}$ is conjugate to $\left(\begin{array}{cc}\left.\kappa_{\mathrm{cyc}}^{-1}\right|_{I_{p}} & 0 \\ * & *\end{array}\right)$,

(3) $\operatorname{det} \rho=\tau \kappa_{\mathrm{cyc}}^{k-3}$ is odd, where $\tau$ is a finite order character and $k \geq 2$.

Then $\rho$ comes from a modular form.

Remark 5.2.3. Our conventions for modular forms and their Galois representations follow those of FK12 and differ slightly from those of SW99. For us, the phrase $\rho$ comes from a modular form means that $\rho$ is isomorphic to $H \otimes_{\mathfrak{h}} F$, where the map $\mathfrak{h} \rightarrow F$ is induced by a cuspidal eigenform $f$ with coefficients in $F$. By [FK12, 1.2.9, 1.5.8], we see that if $\rho$ is modular, then the weight of $f$ must be the integer $k$ of (3).

The representation $\rho$ comes from a modular form in our sense if and only if $\rho \otimes \kappa_{\text {cyc }}$ comes from a modular form in the sense of SW99. With this in mind, the theorem is a restatement of the theorem stated in [SW99, p. 6].

We can give a new proof of this result in certain cases, following directly from Theorem 4.2.8, Among the additional restrictions, the serious ones are:

(iii) that $\rho$ is a lift of "minimal level",

(iv) that a Vandiver conjecture type condition holds for the relevant isotypic parts of the class group.

Now fix $\rho$ and $\theta$ as in Theorem 5.2.2 Let $N$ be the prime-to- $p$ part of the conductor of $\theta$, and write $\chi=\omega^{-1} \theta$. We will treat these both as characters of $G_{\mathbb{Q}}$ and as Dirichlet characters of modulus $N p$.

Theorem 5.2.4. In addition to the conditions of Theorem 5.2.2, assume that

(i) $p \nmid \phi(N)$ and $p \geq 5$;

(ii) $\theta$ is ramified at $p$ when $N>1$;

(iii) $\rho$ is ramified only at primes dividing $N p$;

(iv) $X_{\theta}=0$, and if $\chi$ is unramified at $p$, assume also that $X_{\theta^{-1} \omega^{2}}=0$.

Then $\rho$ comes from a modular form.

The proof of Theorem 5.2.4 relies on applying Theorem 4.2.8, In order to apply that theorem, we will need to relate condition (2) of Theorem 5.2 .2 - which is the "ordinary" condition in SW99 - to our ordinary condition on GMA representations and on pseudorepresentations, established in WWE15 and reviewed in \$3. Note that condition (1) implies condition (b) of \$2.1, so our definition of ordinary applies in this situation. We will see that either $\rho$ is ordinary in our sense or $\chi$ is unramified at $p$ and $\rho \otimes \chi$ is ordinary in our sense.

The following lemma is standard, following from Clifford theory. We provide a proof for lack of a reference.

Lemma 5.2.5. Given any $\nu$ satisfying the conditions of Theorem $5.2 .2,\left.\nu\right|_{G_{p}}$ is reducible. Moreover, there is a unique quotient character $\alpha$ of $\left.\nu\right|_{G_{p}}$ such that $\left.\alpha\right|_{I_{p}}=$ $\left.\kappa_{\text {cyc }}^{-1}\right|_{I_{p}}$.

Proof. Let $\alpha_{1}=\left.\kappa_{\text {cyc }}^{-1}\right|_{I_{p}}$ and $\alpha_{2}=\left.\tau \kappa_{\text {cyc }}^{k-2}\right|_{I_{p}}$. By conditions (2)-(3) of Theorem 5.2 .2 , we have $\left.\nu\right|_{I_{p}} ^{s s} \simeq \alpha_{1} \oplus \alpha_{2}$, and $\alpha_{1}$ is a quotient of $\left.\nu\right|_{I_{p}}$. Since $k \geq 2$ and $\tau$ has finite 
order, we see that $\alpha_{1}$ and $\alpha_{2}$ are distinct. Moreover, since they are restrictions of distinct characters of $G_{p}$, they are not Frobenius conjugate.

The result now follows from Clifford theory, which states that the restriction of an irreducible representation to a normal subgroup is semi-simple and consists of conjugate representations. Indeed, when $\left.\nu\right|_{I_{p}}$ is indecomposable, $\left.\nu\right|_{G_{p}}$ must be reducible and indecomposable, and we let $\alpha$ be the quotient character of $\left.\nu\right|_{G_{p}}$. We see that $\left.\alpha\right|_{I_{p}}=\alpha_{1}$. Otherwise, $\left.\nu\right|_{I_{p}}$ is semi-simple, and since $\alpha_{1}$ and $\alpha_{2}$ are not conjugate, $\left.\nu\right|_{G_{p}}$ must be reducible and semi-simple. We let $\alpha$ be the summand of $\left.\nu\right|_{G_{p}}$ satisfying $\left.\alpha\right|_{I_{p}}=\alpha_{1}$.

We note that our definition of ordinary in Definition 3.4.1 depends on the residual pseudorepresentation. Below, we will sometimes write " $\rho$ is ordinary" to mean " $\rho$ is ordinary as a deformation of $\psi(\bar{\rho})$ ". Let $\bar{D}=\psi\left(\omega^{-1} \oplus \theta^{-1}\right)$ and let $\bar{D}^{\prime}=$ $\psi\left(\omega^{-1} \oplus \theta^{\prime-1}\right)$, where $\theta^{\prime}=\omega \chi^{-1}=\omega^{2} \theta^{-1}$. We are now ready to prove the following.

Lemma 5.2.6. Let $\rho$ be as in Theorem 5.2 .2 and also satisfy condition (iii) of Theorem 5.2.4. If $\chi$ is ramified at $p$, then $\rho$ is an ordinary deformation of $\bar{D}$. If $\chi$ is unramified at $p$, then either $\rho$ is an ordinary deformation of $\bar{D}$ or $\rho \otimes \chi$ is an ordinary deformation of $\bar{D}^{\prime}$.

Proof. We will use the criterion for a representation to be ordinary given in Example 3.4.3. By Lemma 5.2.5, we see that $\left.\rho\right|_{G_{p}}$ is reducible. We will label the JordanHölder factors in two different ways. First we write $\left.\rho\right|_{G_{p}} ^{s s}=\alpha_{1} \oplus \alpha_{2}$ with $\left.\alpha_{1}\right|_{I_{p}}=$ $\left.\kappa_{\text {cyc }}^{-1}\right|_{I_{p}}$. By the same lemma, we see that $\alpha_{1}$ is a quotient of $\left.\rho\right|_{G_{p}}$.

We also write $\left.\rho\right|_{G_{p}} ^{s s}=\alpha_{a} \oplus \alpha_{d}$ with $\bar{\alpha}_{a}=\omega^{-1}$ and $\bar{\alpha}_{d}=\theta^{-1}$. (The labeling is meant to reflect the coordinate labels $\left(\begin{array}{ll}a & b \\ c & d\end{array}\right)$ of a $2 \times 2$-matrix and the ordering convention of (3.1.1).) We see that if $\alpha_{1}=\alpha_{a}$, then $\alpha_{1}$ is a quotient character such that $\bar{\alpha}_{1}=\omega^{-1}$ and $\left.\alpha_{1}\right|_{I_{p}}=\left.\kappa_{\text {cyc }}^{-1}\right|_{I_{p}}$, so $\rho$ is ordinary by Example 3.4.3. Moreover, if $\chi$ is ramified at $p$, we see that $\left.\bar{\alpha}_{a}\right|_{I_{p}} \neq\left.\bar{\alpha}_{d}\right|_{I_{p}}$, and so, since $\left.\bar{\alpha}_{1}\right|_{I_{p}}=\left.\bar{\alpha}_{a}\right|_{I_{p}}$, we must have $\alpha_{1}=\alpha_{a}$.

Now assume that $\alpha_{1}=\alpha_{d}$ (and so $\chi$ is unramified at $p$ ), and let $\rho^{\prime}=\rho \otimes \chi$. Since $\chi$ is unramified at $p$, one can verify that $\rho \otimes \chi$ satisfies the conditions of Theorem 5.2.2. We apply Lemma 5.2.5 to $\rho^{\prime}$, and we get $\left.\rho^{\prime}\right|_{G_{p}} ^{s s}=\alpha_{1}^{\prime} \oplus \alpha_{2}^{\prime}$ with $\alpha_{1}^{\prime}$ a quotient and $\left.\alpha_{1}^{\prime}\right|_{I_{p}}=\left.\kappa_{\text {cyc }}^{-1}\right|_{I_{p}}$. Since $\chi$ is unramified at $p$, we must have $\alpha_{1}^{\prime}=\alpha_{1} \chi$. We also write $\left.\rho^{\prime}\right|_{G_{p}} ^{s s}=\alpha_{a}^{\prime} \oplus \alpha_{d}^{\prime}$ with $\bar{\alpha}_{a}^{\prime}=\omega^{-1}=\theta^{-1} \chi$ and $\bar{\alpha}_{d}^{\prime}=\theta^{\prime-1}=\omega^{-1} \chi$. We see that $\alpha_{a}^{\prime}=\alpha_{d} \chi$. Hence we have

$$
\alpha_{1}^{\prime}=\alpha_{1} \chi=\alpha_{d} \chi=\alpha_{a}^{\prime},
$$

so $\rho^{\prime}$ is ordinary by Example 3.4 .3

The following lemma prepares us to apply Theorem 4.2.8 to prove Theorem 5.2.4.

Lemma 5.2.7. Assume that $\theta, N$, and $p$ satisfy the conditions imposed by Theorems 5.2 .2 and 5.2.4. Then $\theta, N$, and $p$ satisfy the running assumptions of $\$ 2.1$, Also, if $\chi$ is unramified at $p$, then $\theta^{\prime}=\omega^{2} \theta^{-1}$ satisfies these running assumptions.

Proof. The conditions $p \nmid N \phi(N)$ and $p \geq 5$ imposed in $\$ 2.1$ are implied by condition (i) of Theorem 5.2.4 and the definition of $N$ as the tame level of $\theta$.

As we have already noted, $\theta$ and $\theta^{\prime}$ satisfy condition (1) of Theorem 5.2.2, which is equivalent to condition (b) of $\$ 2.1$ Thus it remains to verify conditions (a) and (c). 
Suppose that $N=1$. We claim that the existence of $\rho$ implies that $\theta$ cannot be 1 or $\omega^{2}$. Indeed, in these cases, $\chi=\omega^{ \pm 1}$, and Stickelberger's theorem implies that $X_{\chi}=0$ (see e.g. Was82, Prop. 6.16 and Thm. 6.17, p. 102], and note that $B_{2}=\frac{1}{6}$ ). However, Rib76, Prop. 2.1] implies that the irreducible representation $\rho$ leaves stable a lattice such that the resulting residual representation $\bar{\rho}$ is not diagonalizable and has $\omega^{-1}$ as a subrepresentation. Moreover, because $\rho$ satisfies the conditions of Theorem 5.2.2, and since $\chi$ is ramified at $p$, the argument of Lemma 5.2.6 implies that $\left.\bar{\rho}\right|_{G_{p}}$ has $\omega^{-1}$ as a quotient representation. This implies that $\bar{\rho}$ is a non-trivial extension of $\theta^{-1}$ by $\omega^{-1}$ that is split upon restriction to $G_{p}$. This extension gives rise to a non-zero element of $X_{\chi} \otimes_{\Lambda} \mathbb{F}_{p}$, so $X_{\chi} \neq 0$, a contradiction. Hence, if $N=1$, then $\theta \neq 1$ and $\theta$ is primitive (so (a) is true), and $\theta \neq \omega^{2}$ (so (c) is true).

Now suppose $N>1$, making (c) satisfied. Assumption (i) implies that our running assumption that $p \nmid N \phi(N)$ is satisfied. We have that $\theta$ is primitive of modulus either $N$ or $N p$ by definition of $N$. Assumption (ii) rules out the case that $\theta$ is primitive of modulus $N$, so (a) holds.

When $\chi$ is unramified at $p$, we wish to show that $\theta^{\prime}$ satisfies the running assumptions. Since $\chi$ is odd, the assumption that $\chi$ is unramified at $p$ implies that $N>1$. As $\theta^{\prime}=\omega^{2} \theta^{-1}$, the conductor of $\theta^{\prime}$ is either $N$ or $N p$. Since $\chi^{\prime}=\chi^{-1}$ is unramified at $p$, this implies that $\theta^{\prime}=\chi^{\prime} \omega$ is ramified at $p$, making its conductor $N p$ and satisfying assumption (a).

Proof of Theorem 5.2.4. In Lemma 5.2.7 we checked that the running assumptions of the paper about $\theta, \theta^{\prime}, N$, and $p$ are satisfied. By Lemma 5.2.6, we can break the proof into two cases.

Case 1 ( $\rho$ is ordinary). We consider the case where $\rho$ is ordinary. In this case $\psi(\rho)$ is an ordinary pseudorepresentation, because $\rho$ is an ordinary (GMA) representation inducing it. Therefore there is a unique map $\nu: R_{\bar{D}}^{\text {ord }} \rightarrow F$ corresponding to $\psi(\rho)$. Assumption (iv) allows us to apply Theorem 4.2.8 so that $\phi: R_{\bar{D}}^{\text {ord }} \stackrel{\sim}{\rightarrow} \mathfrak{H}$. Then the ordinary $p$-adic modular eigenform $f$ determined by $\nu \circ \phi^{-1}: \mathfrak{H} \rightarrow F$ satisfies $\psi\left(\rho_{f}\right)=\psi(\rho)$, which implies $\rho_{f} \simeq \rho$ since $\rho$ is irreducible. Condition (3) implies that this modular form has weight $k \in \mathbb{Z}_{\geq 2}$; consequently, $f$ is classical by [Hid86, Thm. I], and $\rho$ is modular.

Case 2 ( $\chi$ is unramified at $p$ and $\rho^{\prime}$ is ordinary). We want to apply Theorem 4.2.8 to $\bar{D}^{\prime}$ now. Assumption (iv) allows us to apply Theorem 4.2.8, so that there is an isomorphism $\phi^{\prime}: R_{\bar{D}^{\prime}} \stackrel{\sim}{\rightarrow} \mathfrak{H}^{\prime}$, where $\mathfrak{H}^{\prime}$ is $\mathfrak{H}$ for $\theta^{\prime}$. Then we have a unique map $\nu^{\prime}: R_{\bar{D}^{\prime}}^{\text {ord }} \rightarrow F$ corresponding to $\psi\left(\rho^{\prime}\right)$, and the rest of the argument is the same as above.

Remark 5.2.8. We see in the proof that for each $\rho$, only one of the two groups $X_{\theta}$ and $X_{\theta^{-1} \omega^{2}}$ must be zero. Moreover, if $\chi$ is ramified at $p$, we can be certain that it is $X_{\theta}$ that must be 0 .

Before SW99, Skinner and Wiles gave a different proof of the modularity of $\rho$ under different hypotheses [SW97]. Among their assumptions is that $X_{\chi^{-1}}=0$. This is equivalent to $\mathfrak{X}_{\theta}=0$ by the reflection principle, and so it is a much stronger assumption than our assumption that $X_{\theta}=0$. In this way, Theorem 5.2.4 may be seen as an improvement of the method of [SW97. 


\section{ACKNOWLEDGMENTS}

The authors would like to thank Romyar Sharifi and Eric Urban for helpful conversations. They also thank the referee for many helpful comments and corrections. Both authors would like to recognize the Simons Foundation for support in the form of AMS-Simons travel grants. The first author was supported by the National Science Foundation under the Mathematical Sciences Postdoctoral Research Fellowship No. 1606255.

\section{REFERENCES}

[BC09] Joël Bellaïche and Gaëtan Chenevier, Families of Galois representations and Selmer groups (English, with English and French summaries), Astérisque 324 (2009), xii+314. MR2656025

[BH93] Winfried Bruns and Jürgen Herzog, Cohen-Macaulay rings, Cambridge Studies in Advanced Mathematics, vol. 39, Cambridge University Press, Cambridge, 1993. MR 1251956

[dSRS97] Bart de Smit, Karl Rubin, and René Schoof, Criteria for complete intersections, Modular forms and Fermat's last theorem (Boston, MA, 1995), Springer, New York, 1997, pp. 343-356. MR 1638484

[FK12] Takako Fukaya and Kazuya Kato, On conjectures of Sharifi. Preprint, 2012.

[FKS14] Takako Fukaya, Kazuya Kato, and Romyar Sharifi, Modular symbols in Iwasawa theory, Iwasawa theory 2012, Contrib. Math. Comput. Sci., vol. 7, Springer, Heidelberg, 2014, pp. 177-219. MR 3586813

[FW79] Bruce Ferrero and Lawrence C. Washington, The Iwasawa invariant $\mu_{p}$ vanishes for abelian number fields, Ann. of Math. (2) 109 (1979), no. 2, 377-395, DOI 10.2307/1971116. MR.528968

[Hid86] Haruzo Hida, Galois representations into $\mathrm{GL}_{2}\left(\mathbf{Z}_{p}[[X]]\right)$ attached to ordinary cusp forms, Invent. Math. 85 (1986), no. 3, 545-613, DOI 10.1007/BF01390329. MR848685

[Jan89] Uwe Jannsen, Iwasawa modules up to isomorphism, Algebraic number theory, Adv. Stud. Pure Math., vol. 17, Academic Press, Boston, MA, 1989, pp. 171-207. MR.1097615

[LS13] Meng Fai Lim and Romyar T. Sharifi, Nekovár duality over p-adic Lie extensions of global fields, Doc. Math. 18 (2013), 621-678. MR.3084561

[Nek06] Jan Nekovář, Selmer complexes (English, with English and French summaries), Astérisque 310 (2006), viii+559. MR2333680

[NSW08] Jürgen Neukirch, Alexander Schmidt, and Kay Wingberg, Cohomology of number fields, 2nd ed., Grundlehren der Mathematischen Wissenschaften [Fundamental Principles of Mathematical Sciences], vol. 323, Springer-Verlag, Berlin, 2008. MR2392026

[Oht05] Masami Ohta, Companion forms and the structure of p-adic Hecke algebras, J. Reine Angew. Math. 585 (2005), 141-172, DOI 10.1515/crll.2005.2005.585.141. MR2164625

[Rib76] Kenneth A. Ribet, A modular construction of unramified p-extensions of $Q\left(\mu_{p}\right)$, Invent. Math. 34 (1976), no. 3, 151-162, DOI 10.1007/BF01403065. MR0419403

[Sha07] Romyar T. Sharifi, Iwasawa theory and the Eisenstein ideal, Duke Math. J. 137 (2007), no. 1, 63-101, DOI 10.1215/S0012-7094-07-13713-X. MR2309144

[Sha09] Romyar T. Sharifi, Cup products and L-values of cusp forms, Pure Appl. Math. Q. 5 (2009), no. 1, 339-348, DOI 10.4310/PAMQ.2009.v5.n1.a10. MR2520463

[Sha11] Romyar Sharifi, A reciprocity map and the two-variable p-adic L-function, Ann. of Math. (2) 173 (2011), no. 1, 251-300, DOI 10.4007/annals.2011.173.1.7. MR2753604

[SW97] C. M. Skinner and A. J. Wiles, Ordinary representations and modular forms, Proc. Nat. Acad. Sci. U.S.A. 94 (1997), no. 20, 10520-10527, DOI 10.1073/pnas.94.20.10520. MR 1471466

[SW99] C. M. Skinner and A. J. Wiles, Residually reducible representations and modular forms, Inst. Hautes Études Sci. Publ. Math. 89 (1999), 5-126 (2000). MR.1793414

[Wak15a] Preston Wake, Eisenstein Hecke algebras and conjectures in Iwasawa theory, Algebra Number Theory 9 (2015), no. 1, 53-75, DOI 10.2140/ant.2015.9.53. MR3317761 
[Wak15b] Preston Wake, Hecke algebras associated to $\Lambda$-adic modular forms, J. Reine Angew. Math. 700 (2015), 113-128, DOI 10.1515/crelle-2013-0017. MR3318512

[Was82] Lawrence C. Washington, Introduction to cyclotomic fields, Graduate Texts in Mathematics, vol. 83, Springer-Verlag, New York, 1982. MR718674

[WE15] Carl Wang-Erickson, Algebraic families of Galois representations and potentially semistable pseudodeformation rings. To appear in Math. Ann., https://doi.org/10.1007/ s00208-017-1557-8, 2015.

[Wei94] Charles A. Weibel, An introduction to homological algebra, Cambridge Studies in Advanced Mathematics, vol. 38, Cambridge University Press, Cambridge, 1994. MR 1269324

[Wi195] Andrew Wiles, Modular elliptic curves and Fermat's last theorem, Ann. of Math. (2) 141 (1995), no. 3, 443-551, DOI 10.2307/2118559. MR.1333035

[WWE15] Preston Wake and Carl Wang-Erickson, Pseudo-modularity and Iwasawa theory. To appear in Amer. J. Math., arXiv:1505.05128v3 [math.NT], 2015.

Department of Mathematics, University of California los Angeles, Box 951555, Los Angeles, California 90095-1555

Email address: wake@math.ucla.edu

Department of Mathematics, Imperial College London, London SW7 2AZ, United KINGDOM

Email address: c.wang-erickson@imperial.ac.uk 